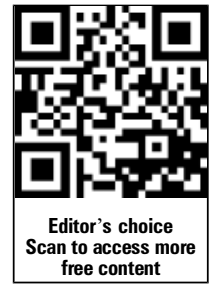

- An additional

supplementary appendix is published online only. To view this file please visit the journal online (http://dx.doi.org/10. 1136/oemed-2012-100924)

${ }^{1}$ Epidemiology of Functioning and Disability, Swiss Paraplegic Research, Nottwil, Lucerne, Switzerland

${ }^{2}$ Department of Health

Sciences and Health Policy, University of Lucerne, Lucerne, Switzerland

${ }^{3}$ International Centre for Life Course Studies in Society and Health, Department of Primary Care and Public Health, Imperial College London, London, UK

${ }^{4}$ Department of Medical Sociology, Heinrich Heine University of Duesseldorf, Duesseldorf, Germany

\section{Correspondence to}

Dr Jan D Reinhardt, Epidemiology of Functioning and Disability, Swiss Paraplegic Research, Guido A Zäch Str 4, Nottwil, Lucerne 6207. Switzerland

jan.reinhardt@paranet.ch

Received 16 May 2012

Revised 5 October 2012

Accepted 12 November 2012

Published Online First

13 December 2012
To cite: Reinhardt JD, Wahrendorf M, Siegrist J. Occup Environ Med 2013;70:156-163.

\title{
Socioeconomic position, psychosocial work environment and disability in an ageing workforce: a longitudinal analysis of SHARE data from 11 European countries
}

\author{
Jan D Reinhardt, ${ }^{1,2}$ Morten Wahrendorf, ${ }^{3}$ Johannes Siegrist ${ }^{4}$
}

\begin{abstract}
Objectives Prevention of disability in the ageing workforce is essential for sustaining economic growth in Europe. In order to provide information on entry points for preventive measures, it is important to better understand sociodemographic, socioeconomic and workrelated determinants of disability in older employees. We aimed to test the hypothesis that low socioeconomic position and exposure to a stressful psychosocial work environment at baseline contribute to later disability. We further assumed that the association of socioeconomic position with disability is partly mediated by exposure to adverse working conditions.

Methods We studied longitudinal data from the first two waves of the Survey on Health, Ageing and Retirement in Europe comprising 11 European countries. Sociodemographic, socioeconomic and work-related factors (low control, effort-reward imbalance) and baseline disability of 2665 male and 2209 female employees aged between 50 and 64 years were used to predict disability 2 years later. Following the International Classification of Functioning (ICF), disability was subdivided into the components 'impairment' and 'restriction in activities and participation'. Two multilevel Poisson regressions were fitted to the data.

Results After adjusting for baseline disability and

\section{What the paper adds}

- Previous investigations demonstrated a social gradient of disability in older populations, but were restricted to single countries or cohorts, were mostly cross-sectional, or did not use a comprehensive measure of disability.

- In this study, we followed the guidelines of the International Classification of Functioning and used multiple variables to construct and validate two different disability indices (1. impairment; 2 . restrictions in activity and participation) in the frame of a longitudinal cross-country study.

- Our findings based on employees aged 50-64 years from 11 European countries demonstrate a social gradient of both indices of disability which is in part mediated by adverse psychosocial working conditions.

- Investment into reduction of psychosocial work stress, in particular among employees in lower socioeconomic positions, is a relevant entry point of policies that aim at maintaining work ability in the ageing workforce.
\end{abstract} relevant confounding variables, low socioeconomic position and chronic stress at work exerted significant effects on disability scores 2 years later. We found some support for the hypothesis that the association of socioeconomic position with disability is partly mediated by work stress.

Conclusions Investing in reduction of work stress and reducing social inequalities in health functioning are relevant entry points of policies that aim at maintaining work ability in early old age.

\section{INTRODUCTION}

European populations are ageing faster than populations in many other parts of the world. ${ }^{1}$ Despite a continued increase in healthy life expectancy, a substantial proportion of 'people in early old age' (approx. 50-64 years) in Europe suffer from some degree of impairment which often limits their work capability. ${ }^{2}$ In fact, reduced health functioning and disability are major determinants of involuntary early exit from the labour market. ${ }^{3}{ }^{4}$ In view of the strong economic need for extending labour market participation of older people in European countries, ${ }^{2}$ it is important to know more precisely the sociodemographic, socioeconomic and workrelated characteristics of older men and women whose work capability is limited due to impairment or disability. Such knowledge may provide useful information on entry points for preventive measures within and beyond occupational settings.

Previous investigations documented a social gradient of disability prevalence in early old age, leaving men and women in lower socioeconomic positions at higher risk. ${ }^{5-10}$ However, given the bidirectional pathways between health functioning and socioeconomic position, including employment status, in a life course perspective, ${ }^{3}$ a clear-cut interpretation of this association is difficult. Moreover, measures of socioeconomic position in terms of occupational position or earnings are often confounded by distinct occupational exposures (eg, a high prevalence of jobs with heavy physical demands among men with low occupational position), which may trigger early onset of disability. ${ }^{11}$ It is therefore important 
to disentangle these effects and to test their robustness in a large dataset which offers opportunities of adjusting for relevant confounders.

In this study, we set out to test associations of sociodemographic, socioeconomic and work-related factors with disability in a prospective study design, using strictly comparable indicators, based on a large sample of male and female employees in early old age from 11 European countries. More specifically, we test the hypothesis that low socioeconomic position and exposure to a stressful psychosocial work environment (assessed at wave 1) contribute to later disability (assessed 2 years later at wave 2). Furthermore, we claim that the association of socioeconomic position with disability is partly mediated by exposure to a stressful psychosocial work environment, as measured by core components of two established theoretical models: the demand-control ${ }^{12}$ and the effort-reward imbalance (ERI) ${ }^{13}$ models.

The demand-control (or 'job strain') model defines work stress in terms of a distinct job task profile where jobs defined by high quantitative demands in combination with low decision latitude or low task control are stressful. ${ }^{12} \mathrm{~A}$ complementary model, ERI, focuses on the work contract and the principle of social reciprocity lying at its core. ${ }^{13}$ Rewards received in return for efforts spent at work include money, esteem and career opportunities (promotion prospects, job security). The model asserts that lack of reciprocity (high effort in combination with low reward) occurs frequently in modern economies and generates strong negative emotions and psychobiological stress responses with adverse long-term effects on health.

A second aim of this study concerns the development of indices of core dimensions of disability, taking note of a more comprehensive approach to this notion, as documented in basic work resulting in the International Classification of Functioning, Disability and Health (ICF). ${ }^{14}$ Disability is no longer interpreted as a fixed attribute of an individual, but rather as a dynamic continuum of experiences at different levels (bodily impairment, restrictions in activity and social participation). As disability results from an interaction of vulnerable individuals with a broad range of environmental factors including working conditions, ${ }^{15}{ }^{16}$ it seems mandatory to assess this condition in a multidimensional, quantitative frame of analysis. At least two crucial components need to be distinguished, the level of bodily impairment and the level of restriction in activities and social participation, since 'people with the same impairment can experience very different types and degrees of restriction, depending on the context'. ${ }^{16}$ A person with an amputation, may for instance, not be restricted in activities and participation when she is provided with appropriate prostheses and supports. Another reason for this distinction is related to their potentially different role in predicting morbidity and mortality. In a recent study of older British women, it was observed that restrictions in participation, and restrictions in complex activities, were associated with increased risk of mortality, but no such effect was found for impairment in the fully adjusted models. ${ }^{17}$ Therefore, embedded in the framework of ICF, we aim at developing a comprehensive continuous measure of core dimensions of disability, based on available data.

\section{METHODS}

\section{Data source}

Data were obtained from the first two waves of the 'Survey of Health, Ageing and Retirement in Europe' (SHARE, Release 2.5). SHARE is the first longitudinal research project comparing data on working conditions, retirement, health and well-being, and social position among people aged 50 years and older in a variety of European countries. ${ }^{18}$ Data from the two first waves (wave 1: 2004-2005; wave 2: 2006-2007) include 11 European countries (Sweden, SE; Denmark, DK; Germany, DE; The Netherlands, NL; Belgium, BE; France, FR; Switzerland, $\mathrm{CH}$; Austria, AT; Italy, IT; Spain, ES: Greece, GR). Data collection is based on probability household samples where all people above 50 years of age, plus their (possibly younger) partners, were interviewed using Computer Assisted Personal Interviews. Due to different institutional settings in the participating countries, the sampling was either drawn as a stratified simple random selection from the national population register (Denmark and Sweden), or as a multistage sampling using regional and local registers (Germany, Italy, Spain, France, The Netherlands) or telephone directories (Greece, Austria, Switzerland). In SHARE, information is available for 28517 respondents in wave 1 , where the country average of household response rate is $60.6 \%$ for the total sample, ranging from $38.8 \%$ in Switzerland to $79.2 \%$ in France (rates above $50 \%$ in eight countries). In wave 2, 18742 of these individuals remain in the sample, with an attrition rate of $27.9 \%$. $^{35}$ Because we were interested in associations between working conditions in late midlife in wave 1 and disability in wave2, we excluded people not aged 50-64 years at wave 1 $(\mathrm{n}=8196)$, and those reporting no employment at baseline $(n=12485)$ from the longitudinal sample. Moreover, we conducted a complete case analyses, thus excluding respondents with missing data on any of the variables $(n=2636)$. This results in a final sample size of 4864 respondents. In order to compensate for unit non-response, and for attrition between the first and the second waves, calibrated longitudinal weights were applied. These weights are defined for the longitudinal sample only and are calculated for each country separately (see ref. 19 for details on methodological issues in SHARE).

\section{Measurement}

Disability

Based on ICF and its definition of disability, we divided between two indices of disability: one measuring impairment and another measuring restriction in activity and participation (A\&P). ${ }^{20}$ Accordingly, all SHARE modules were screened for questions related to disability, and respective items were linked to the two dimensions using established linking rules. ${ }^{2021}$ Since most original items were dichotomous, we dichotomised the remaining items, such that one category was indicative of having at least a moderate problem in its respective domain. As a next step, we applied a principal component analysis to evaluate the chosen items based on wave 1 data (see table 1 ). In the results, two factors with eigenvalues above 2.0 were extracted confirming the assumed dimensions of disability. However, two candidate items for the A\&P factor did not load as expected ('using a map in a strange place' had a loading below 0.1 and the overall activity limitation item loaded on the impairment factor) and were excluded from further analysis. Not considering the dropped items, the two factors explained around $34 \%$ of the variance of all items, and $45 \%$ of the A\&P, and $23 \%$ of the impairment items were explained by the two-factor solution. Next, we performed a confirmatory factor analysis for item responses at wave 2 (2006) (table 1) using the STATA module confa. $^{22}$ The first item of the respective index was used as scalar (loading set to 1). Results show that our index solution meets the statistical criteria. However, one item on the impairment factor and two items on the A\&P restrictions factor displayed marginal loadings only, probably due to their low prevalence, but were, nevertheless, included in the final index solution. In 
Table 1 Construction of sum indices for impairment and A\&P restrictions

\begin{tabular}{|c|c|c|c|c|c|}
\hline Construct & Variable & Measurement & Coding for 'yes' & PCA* & CFAt \\
\hline \multirow[t]{12}{*}{ Impairment } & Symptoms load & Two or more symptoms & 1 & 0.16 & 1 \\
\hline & Problems with biting & Unable to bite on hard food & 1 & 0.12 & 0.15 \\
\hline & Problems with seeing & Seeing is fair or worse (either distance or reading) & 1 & 0.14 & 0.22 \\
\hline & Problems with hearing & Hearing is fair or worse & 1 & 0.10 & 0.21 \\
\hline & Mobility & $\begin{array}{l}\text { One or more mobility, arm function and } \\
\text { fine motor limitations }\end{array}$ & 1 & 0.18 & 0.9 \\
\hline & $\begin{array}{l}\text { Orientation to date, month, } \\
\text { year and day of week }\end{array}$ & Less than good & 1 & 0.15 & 0.07 \\
\hline & Mathematical performance & Score 1 or 2 (range $1-5$ ) & 1 & 0.18 & 0.15 \\
\hline & Grip strength & Male: <37/female: $<21$ & 1 & 0.15 & 0.19 \\
\hline & Depressive symptoms & Eurodcat & 1 & 0.16 & 0.53 \\
\hline & Verbal fluency score & Less than 15 & 1 & 0.23 & 0.11 \\
\hline & Ten words list & Three or less & 1 & 0.24 & 0.12 \\
\hline & Delay ten words list & Two or less & 1 & 0.25 & 0.15 \\
\hline \multicolumn{6}{|l|}{ Range sum index } \\
\hline \multirow[t]{12}{*}{ A\&P restrictions } & Reported problems with... & Dressing, including putting on shoes and socks & 1 & 0.24 & 1 \\
\hline & & Walking across a room & 1 & 0.34 & 0.19 \\
\hline & & Bathing or showering & 1 & 0.29 & 0.40 \\
\hline & & Eating, such as cutting up your food & 1 & 0.29 & 0.07 \\
\hline & & Getting in and out of bed & 1 & 0.27 & 0.54 \\
\hline & & Using the toilet, including getting up or down & 1 & 0.30 & 0.22 \\
\hline & & Preparing a hot meal & 1 & 0.22 & 0.19 \\
\hline & & Shopping for groceries & 1 & 0.26 & 0.63 \\
\hline & & Making telephone calls & 1 & 0.19 & 0.06 \\
\hline & & Taking medications & 1 & 0.26 & 0.11 \\
\hline & & Doing work around the house or garden & 1 & 0.24 & 1.14 \\
\hline & & $\begin{array}{l}\text { Managing money, such as paying bills and } \\
\text { keeping track of expenses }\end{array}$ & 1 & 0.27 & 0.09 \\
\hline Range sum index & & & $0-12$ & & \\
\hline
\end{tabular}

*Principal component analysis (PCA) with 8615 cases from the selected countries for 2004; numbers are loadings on respective components, two components impairment and A\&P restriction with eigenvalues $>2$ extracted.

tConfirmatory factor analysis (CFA) for the final sample $(n=4864)$. Numbers indicate loadings on component impairment and A\&P restriction; the first items were used as scalars.

case of A\&P restrictions, it should be noted that the included items turned out to be part of two other existing scales (ADL-scale, IADL-scale). Yet, not all items of these two latter scales were included, and instead of dichotomising both scales into 'No limitations' versus 'One or more limitations' (as usually done), we created one single scale with additional information on the number of A\&P limitations. We constructed sum indices for impairment and A\&P restrictions ${ }^{21}$ (table 1). Each of those is a count variable ranging from 0 (no reported impairment or $\mathrm{A} \& \mathrm{P}$ restrictions) to 12 (12 reported impairments or A\&P restrictions). We plotted both indices against a Poisson distribution which was confirmed in both cases. Expectedly, the impairment scale showed a high correlation with subjective health (Pearsons $r=0.40)$, while the A\&P scale showed a lower correlation of $r=0.17$. Both scales were correlated with $r=0.2$. This demonstrates convergent as well as divergent validity, that is, the scales seem to measure different constructs.

\section{Working conditions}

Quality of work was assessed by a short battery of items derived from (a) the Job Content Questionnaire measuring the demandcontrol model ${ }^{23}$ and (b) from the ERI model questionnaire ${ }^{24}$ (see online supplementary appendix 1). The psychometric properties of both questionnaires were previously tested. ${ }^{24}$ Given the constraints of a multidisciplinary approach in SHARE project, the inclusion of the full questionnaires was not possible. Thus, items with best psychometric properties in their respective scales were selected. With regard to the first model, the measurement was restricted to the control dimension. This decision was based on evidence that the predictive power of 'control' by far exceeded the power of 'demand', and that tests of the interaction term 'demand' $\times$ 'control' had produced inconsistent results. ${ }^{25}$ Low control at work was measured by the sum score of two Likert-scale items ranging from 2 to 8 , with higher scores indicating lower control at work. Scores in the upper tertile of each country were defined as representing poor quality of work in terms of low control.

To measure ERI, two out of six items measuring 'effort', and five out of 11 items assessing 'reward' at work were included. For the selected items, all-item total correlations were far beyond the established threshold of $0.30,{ }^{26}$ ranging from 0.93 to 0.81 (uncorrected), and from 0.67 to 0.42 (corrected). 'Effort-reward imbalance' was defined by a ratio of the sum score of the 'effort' items (nominator) and of the sum score of the 'reward' items (adjusted for number of items; denominator). As previous analyses showed that quality of work in terms of this model varies across countries under study in SHARE, tertiles of the ratio were calculated for each country separately. ${ }^{24}$ Participants scoring in the upper tertiles of this ratio of imbalance were considered experiencing poor quality of work.

\section{Additional measures}

Age, gender, income and education were included as additional measures. Income information is based on the total annual 
Table 2 Sample description and mean disability scores and SD according to covariates $(n=4864)$

\begin{tabular}{|c|c|c|c|c|}
\hline Variables & $\begin{array}{l}\text { Prevalence (\%) } \\
\text { (unweighted) }\end{array}$ & $\mathrm{n}$ & $\begin{array}{l}\text { W2* mean } \\
\text { impairment (SD) }\end{array}$ & $\begin{array}{l}\text { W2* mean } \\
\text { A\&P (SD) }\end{array}$ \\
\hline \multicolumn{5}{|l|}{ Sex } \\
\hline Male & 54.6 & 2655 & $1.72(1.69)$ & $0.09(0.51)$ \\
\hline Female & 45.4 & 2209 & $2.01(1.64)$ & $0.06(0.33)$ \\
\hline$p$ Value & & & $<0.001$ & 0.052 \\
\hline \multicolumn{5}{|l|}{ Age group } \\
\hline $50-55$ years & 43.6 & 2123 & $1.68(1.52)$ & $0.08(0.49)$ \\
\hline $55-59$ years & 37.0 & 1798 & $1.95(1.76)$ & $0.07(0.44)$ \\
\hline $60-65$ years & 19.4 & 943 & $2.05(1.84)$ & $0.07(0.29)$ \\
\hline $\mathrm{p}$ Value & & & $<0.001$ & 0.571 \\
\hline \multicolumn{5}{|l|}{ Education } \\
\hline High & 31.0 & 1509 & $1.38(1.38)$ & $0.05(0.24)$ \\
\hline Medium & 36.4 & 1771 & $1.72(1.50)$ & $0.10(0.53)$ \\
\hline Low & 32.6 & 1584 & $2.45(1.95)$ & $0.08(0.46)$ \\
\hline $\mathrm{p}$ Value & & & $<0.001$ & 0.004 \\
\hline \multicolumn{5}{|l|}{ Income } \\
\hline High & 54.1 & 2631 & $1.62(1.51)$ & $0.07(0.45)$ \\
\hline Medium & 30.5 & 1482 & $1.95(1.74)$ & $0.08(0.38)$ \\
\hline Low & 15.4 & 751 & $2.35(1.90)$ & $0.11(0.54)$ \\
\hline$p$ Value & & & $<0.001$ & 0.104 \\
\hline \multicolumn{5}{|l|}{ Low control } \\
\hline Yes & 22.6 & 1099 & $2.32(1.96)$ & $0.11(0.53)$ \\
\hline No & 77.4 & 3765 & $1.73(1.58)$ & $0.07(0.42)$ \\
\hline$p$ Value & & & $<0.001$ & 0.017 \\
\hline \multicolumn{5}{|c|}{ Effort reward imbalance } \\
\hline Yes & 31.3 & 1525 & $2.21(1.82)$ & $0.12(0.60)$ \\
\hline No & 68.7 & 3339 & $1.68(1.58)$ & $0.06(0.35)$ \\
\hline $\mathrm{p}$ Value & & & $<0.001$ & $<0.001$ \\
\hline Overall & 100.0 & 4864 & $1.84(1.68)$ & $0.08(0.44)$ \\
\hline
\end{tabular}

household income composed by the sum of different income components that were assessed in the questionnaire. In case income components were missing, information was obtained through imputation. $^{27}$ To adjust for household size, we divided the value of income in accordance with the OECD equivalent scale, and categorised it into country-specific tertiles based on all available cases in each country (low, medium, high). Education was measured according to the International Standard Classification of Educational Degrees (ISCED-97) that was categorised into 'low education' (preprimary, primary or lower secondary education), 'medium education' (secondary or postsecondary education), and 'high education' (first and second stage of tertiary education). These two indicators are introduced as proxy measures of socioeconomic position, but are analysed separately as they point to different dimensions of social inequality. $^{28}$

\section{Analyses}

As a first step, table 2 gives an overview of the study sample and the core variables. Additionally, associations between disability and all covariates are analysed using analysis of variance. Thereafter, we calculated Poisson regression models to predict disability $^{20}$ in wave 2 . Given the multilevel structure of the data, we estimated a random intercept multilevel model with individuals (level 1) nested within countries (level 2). ${ }^{29}$ This model contains two components: a so-called fixed component that explains systematic variability in the data, and a random component which accounts for unobserved variability between countries (random intercepts for each country). This procedure allows for accurate adjustment for country affiliation. Maximum likelihood estimation is used for parameter estimation. In the Results section, for each one of the two disability outcomes, we display findings based on three consecutive models, all adjusted for age, gender and level of impairment at wave 1 . This procedure allows for exploring to what extent the predicting variables are associated with change in disability between wave 1 and wave $2 .{ }^{30}$ Model 1 investigates the joint effect of income and education on disability. In model 2 , the joint effects of the two work-stress measures are presented. Model 3 includes all variables of the first two models, with the aim of examining potential mediation effects. In respective tables, we present incidence rates ratios together with the level of statistical significance and CIs. Calculations were done using the 'xtmepoisson' procedure in STATA 11.

\section{RESULTS}

\section{Descriptive findings}

In table 2, sample characteristics are given, and mean scores of the two disability factors, impairment and A\&P restrictions, are displayed according to covariates. A total of 2655 men and 
Figure 1 Mean disability scores (in wave 2) according to income (in wave 1, top) and effort-reward imbalance (in wave 1, bottom) across Survey on Health, Ageing and Retirement in Europe countries $(n=4864)$. Note: means are based on weighted data.

Impairment and A\&P across SHARE countries

by Income
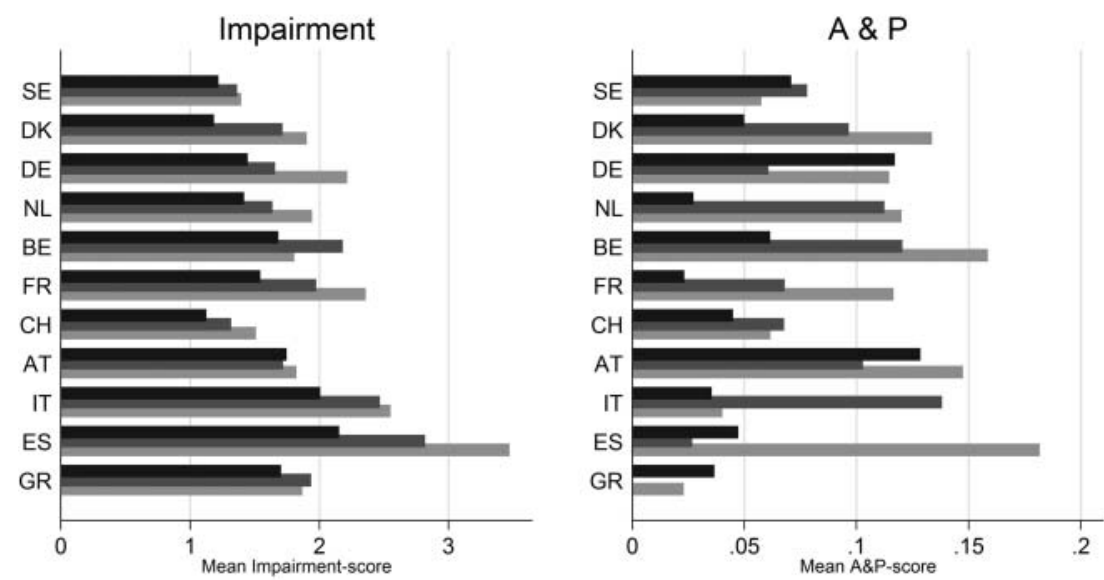

High income

Medium income

Low income

Source: SHARE (release 2.5.0)

Impairment and A\&P across SHARE countries by Effort-Reward Imbalance
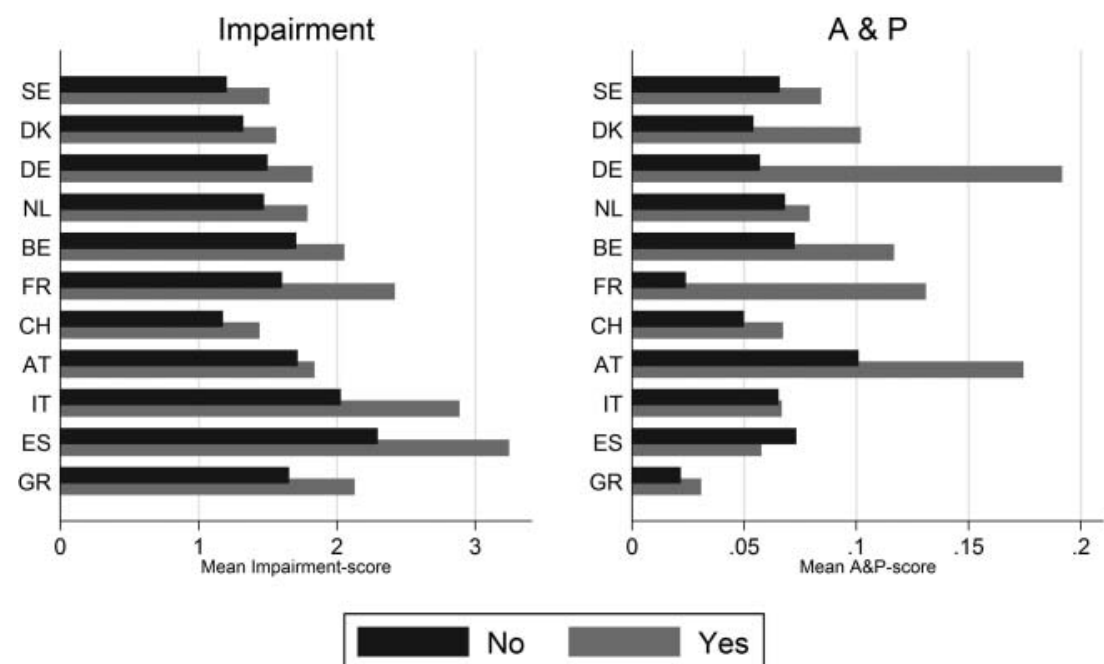

Source: SHARE (release 2.5.0)

2209 women provided full data and were included in the analyses. The age distribution was as follows: $43.6 \%$ were 50-54 years, $37 \%$ were $55-59$ years, and $19.4 \%$ were 60-64 years old. A majority of the participants had medium or low education, and more than half had a high income. With regard to work stress, $22.6 \%$ experienced low control at work and $31.3 \%$ experienced an imbalance between high efforts and low rewards according to our classification.

Concerning the scores of impairment and A\&P restrictions, we first observe that overall levels of A\&P restrictions are much lower than those of impairment scores. Impairment scores varied according to gender (significantly higher values for women), age (higher values for older participants) and socioeconomic position (higher values for people with lower education or lower income). As shown in figure 1 (top), this social gradient is consistent across all countries, using income as an indicator of socioeconomic position. Less clear findings are obvious in case of A\&P restrictions.
As expected, we observe higher scores of the two disability factors among men and women experiencing chronic stress at work in terms of the two work-stress models. Again, relatively consistent trends are observed across the countries under study, as exemplified by measuring work stress in terms of ERI (figure 1, bottom).

\section{Multivariate findings}

In tables 3 and 4, results of multivariate analyses are presented. In both tables, we present findings of the three regression models described in the Methods section, first for impairment, and second for A\&P restrictions.

Significant effects of gender (women), age (older), socioeconomic position (lower), and work stress (both models) on impairment scores were confirmed in multivariate analysis (models 1 and 2). If the indicators of socioeconomic position and the two work-stress models are included simultaneously (model 3), we observe an attenuated effect of the two indicators 
Table 3 Predictors of impairment: results of multilevel Poisson regression models: incidence rate ratios and significance level $(n=4864)$

\begin{tabular}{|c|c|c|c|c|c|c|}
\hline \multirow[b]{3}{*}{ Variables } & \multicolumn{6}{|l|}{ Model } \\
\hline & \multicolumn{2}{|l|}{1} & \multicolumn{2}{|l|}{2} & \multicolumn{2}{|l|}{3} \\
\hline & IRR & $\mathrm{Cl}(95 \%)$ & IRR & $\mathrm{Cl}(95 \%)$ & IRR & $\mathrm{Cl}(95 \%)$ \\
\hline \multicolumn{7}{|l|}{ Sex } \\
\hline \multicolumn{7}{|l|}{ Male } \\
\hline Female & $1.06^{*}$ & 1.01 to 1.11 & $1.06^{*}$ & 1.01 to 1.10 & $1.06 t$ & 1.01 to 1.11 \\
\hline \multicolumn{7}{|l|}{ Age group } \\
\hline \multicolumn{7}{|l|}{$<55$ years } \\
\hline $55-59$ years & $1.07 \dagger$ & 1.02 to 1.12 & $1.07^{*}$ & 1.01 to 1.12 & $1.07 \dagger$ & 1.02 to 1.12 \\
\hline $60-65$ years & $1.11 \ddagger$ & 1.05 to 1.18 & $1.13 \ddagger$ & 1.06 to 1.20 & $1.12 \ddagger$ & 1.05 to 1.19 \\
\hline \multicolumn{7}{|l|}{ Education } \\
\hline \multicolumn{7}{|l|}{ High } \\
\hline Medium & $1.08 t$ & 1.02 to 1.15 & & & $1.07^{*}$ & 1.01 to 1.13 \\
\hline Low & $1.19 \ddagger$ & 1.12 to 1.27 & & & $1.16 \ddagger$ & 1.09 to 1.24 \\
\hline \multicolumn{7}{|l|}{ Income } \\
\hline \multicolumn{7}{|l|}{ High } \\
\hline Medium & $1.07 t$ & 1.02 to 1.12 & & & $1.06^{*}$ & 1.01 to 1.12 \\
\hline Low & $1.11 \ddagger$ & 1.05 to 1.18 & & & $1.10 t$ & 1.03 to 1.17 \\
\hline \multicolumn{7}{|l|}{ Low control } \\
\hline \multicolumn{7}{|l|}{ No } \\
\hline Yes & & & $1.10 \ddagger$ & 1.04 to 1.15 & $1.07 \dagger$ & 1.02 to 1.13 \\
\hline \multicolumn{7}{|l|}{ Effort reward imbalance } \\
\hline \multicolumn{7}{|l|}{ No } \\
\hline Yes & & & $1.12 \ddagger$ & 1.07 to 1.17 & $1.10 \ddagger$ & 1.05 to 1.15 \\
\hline Prior impairment state & $1.24 \ddagger$ & 1.22 to 1.25 & $1.25 \ddagger$ & 1.23 to 1.26 & $1.23 \ddagger$ & 1.22 to 1.25 \\
\hline
\end{tabular}

of socioeconomic position on impairment, pointing to a partial mediation of the association between socioeconomic position and impairment by a high level of work stress. Results are not as consistent in case of A\&P restrictions (table 4). Effects of gender and age are not significant in the multivariate model, whereas the remaining effects correspond to those of impairment.

\section{DISCUSSION}

Based on two summary indices of impairment and restrictions of activities and participation, we tested sociodemographic, socioeconomic and work-related predictors of disability among working men and women of early old age across 11 European countries, using data from two waves of SHARE study. After adjusting for baseline disability and relevant confounding variables, low socioeconomic position and chronic stress at work (low control, ERI) exerted significant effects on disability scores 2 years later. Effects were more consistent for impairment than for $A \& P$ restrictions. In addition, we found some support of the hypothesis that the association of socioeconomic position with disability is partly mediated by work stress (tables 3 and 4; model 3). To our knowledge, no former study analysed these associations in a prospective design comparing data across a variety of European countries. A further innovative aspect of this investigation concerns the construction of linear indices of two core components of a comprehensive concept of human functioning that was developed in the frame of ICF. $^{14}$

Previous studies found similar associations of socioeconomic position with measures of disability, but were restricted to single cohorts or single countries. ${ }^{5-10}$ Huisman, ${ }^{5}$ for instance, found that inequalities were lowest in the oldest age group, particularly among women. However, due to the cross-sectional nature of this study, it cannot be determined if this is an ageing or cohort effect. Moreover, it is unclear if the reported socioeconomic differences in long-term disability are in part due to differential working conditions. Similarly, a few investigations tested prospective associations of job strain ${ }^{3132}$ or ERI $^{31-33}$ with reduced functioning, often measured by the SF-36 or short versions. ${ }^{34}$ Stansfeld ${ }^{31}$ found, for example, that high demands and ERI and negative aspects of close relationships were independent predictors of poor SF-36 functioning. However, other indicators of socioeconomic position, such as income and education, were not analysed. In this study, we add information on the combined effect of socioeconomic position and work strain on two core indicators of disability, and further on a partial mediating role of work stress in this former association.

Several limitations need to be mentioned. First, most items we used for construction of the disability indices were based on self-reported data rather than on functional testing. Therefore, the validity of our indices may be limited, even more so due to potential reporting bias (eg, social desirability). However, graded associations with age and, in case of impairment, consistent variations with gender and socioeconomic position point to a valid assessment. As reporting bias is assumed to vary across countries it is unlikely that it inflates the findings of the total sample. Nevertheless, future studies should aim at integrating more 'objective' functional measures and determining differences between self-reported and observational data. A second limitation concerns the assessment of stressful work with 
Table 4 Predictors of A\&P restrictions: results of multilevel Poisson regression models: incidence rate ratios and significance level ( $n=4864$ )

\begin{tabular}{|c|c|c|c|c|c|c|}
\hline \multirow[b]{3}{*}{ Variables } & \multicolumn{6}{|l|}{ Model } \\
\hline & \multicolumn{2}{|l|}{1} & \multicolumn{2}{|l|}{2} & \multicolumn{2}{|l|}{3} \\
\hline & IRR & $\mathrm{Cl}(95 \%)$ & IRR & $\mathrm{Cl}(95 \%)$ & IRR & $\mathrm{Cl}(95 \%)$ \\
\hline \multicolumn{7}{|l|}{ Sex } \\
\hline \multicolumn{7}{|l|}{ Male } \\
\hline Female & 0.97 & 0.78 to 1.21 & 0.96 & 0.77 to 1.19 & 0.97 & 0.77 to 1.20 \\
\hline \multicolumn{7}{|l|}{ Age group } \\
\hline \multicolumn{7}{|l|}{$<55$ years } \\
\hline $55-59$ years & 1.07 & 0.84 to 1.36 & 1.04 & 0.82 to 1.32 & 1.06 & 0.83 to 1.35 \\
\hline $60-65$ years & 0.99 & 0.73 to 1.33 & 1.01 & 0.75 to 1.36 & 1.00 & 0.74 to 1.35 \\
\hline \multicolumn{7}{|l|}{ Education } \\
\hline \multicolumn{7}{|l|}{ High } \\
\hline Medium & $1.93 \ddagger$ & 1.42 to 2.62 & & & $1.83 \ddagger$ & 1.34 to 2.50 \\
\hline Low & $2.02 \ddagger$ & 1.45 to 2.80 & & & $1.87 \ddagger$ & 1.34 to 2.60 \\
\hline \multicolumn{7}{|l|}{ Income } \\
\hline \multicolumn{7}{|l|}{ High } \\
\hline Medium & $1.34^{*}$ & 1.05 to 1.72 & & & $1.30^{*}$ & 1.01 to 1.67 \\
\hline Low & $1.36^{*}$ & 1.01 to 1.85 & & & 1.31 & 0.97 to 1.78 \\
\hline \multicolumn{7}{|l|}{ Low control } \\
\hline \multicolumn{7}{|l|}{ No } \\
\hline Yes & & & $1.43 \dagger$ & 1.12 to 1.82 & $1.30^{*}$ & 1.02 to 1.66 \\
\hline \multicolumn{7}{|l|}{ Effort reward imbalance } \\
\hline \multicolumn{7}{|l|}{ No } \\
\hline Yes & & & $1.40 \dagger$ & 1.11 to 1.75 & $1.31^{*}$ & 1.04 to 1.64 \\
\hline Prior A\&P restrictions & $1.53 \ddagger$ & 1.44 to 1.63 & $1.55 \ddagger$ & 1.45 to 1.64 & $1.52 \ddagger$ & 1.43 to 1.62 \\
\hline
\end{tabular}

abbreviated scales. A test of the full models using original measures of all respective scales (including 'demand' and 'social support' in the job strain model, and including 'overcommitment' in the ERI model) may reduce the risk of underestimating their effects on prospective disability. Third, we restricted our analyses to the assessment of work stress at wave 1. Additional information on the duration of exposure available from the assessment of work stress at wave 2 could enrich the current analyses. Also, we did not consider data on non-work-related or extracurricular exposures or information on the cause of impairment, for example, injury versus disease. This will be possible with data from SHARE wave 3 that was focused on retrospective assessment of the participants life courses.

Since previous analyses indicate that healthier people were more likely to participate in wave $2,{ }^{35}$ we also cannot rule out some selection bias, where people with low levels of disability are more likely to participate in survey research. However, this may rather lead to an underestimation of disability levels and its association with work stress due to health selection. Furthermore, while our analyses focussed on individual predictors, future research may also explore the role of distinct national disability policies and their interactions with working conditions. $^{2} 36$ Similarly, albeit the conducted multivariate analyses account for country affiliation, future research may invest in more detailed differences between countries and measurement equivalence of our two indices. In this regard, additional explorative principal component analysis by country showed that A\&P items mostly loaded on the same factor which did not hold true for several impairment items.
These limitations are balanced by several strengths. First, the SHARE study applied strictly comparable procedures in data collection based on identical measures and study designs across all countries. ${ }^{19}$ Second, by applying multilevel statistical modelling in the analysis of data from two measurement waves, by including relevant confounding factors in multivariate analysis, and by providing quantitative indices of two relevant dimensions of disability, our results can be considered rather robust, even more so as they are based on a large sample representative of working men and women aged 50-64 years within the respective countries. Third, our analysis is based on two established, theoretically grounded notions of stressful work, lack of control and failed reciprocity between effort and reward. ${ }^{37}$ Both models were previously tested in a range of prospective cohort studies and were shown to contribute to the prediction of stress-related disorders, such as coronary heart disease ${ }^{38}$ and depressive episodes. ${ }^{39}$ Available evidence on health-adverse effects of an adverse psychosocial work environment strengthens the significance of current findings with regard to reduced functioning in terms of impairment and restricted activities and social participation. Furthermore, additional analyses based on SHARE data demonstrate that the experience of stressful work in terms of these models increases the likelihood of intended early retirement. ${ }^{37}$

In conclusion, low socioeconomic position and stressful work are prospectively associated with two core indices of disability in a large cohort of working men and women of early old age from 11 European countries. Investing in good quality of work and reducing social inequalities in health and functioning are relevant entry points of policies that aim at maintaining work ability in early old age. 
Acknowledgements This study was funded by the Swiss Paraplegic Research in Nottwil, Switzerland. MW was supported by a grant from the German Research Foundation (project number: WA 3065/1-1).

This paper uses data from SHARE release 2.5.0, as of May 24, 2011. The SHARE data collection has been primarily funded by the European Commission through the 5th framework programme (project QLK6-CT-2001-00360 in the thematic programme Quality of Life), through the 6th framework programme (projects SHARE-I3, RII-CT-2006-062193, COMPARE, CIT5-CT-2005-028857, and SHARELIFE, CIT4-CT-2006-028812), and through the 7th framework programme (SHARE-PREP, 211909 and SHARE-LEAP, 227822). Additional funding from the US National Institute on Aging (U01 AG09740-13S2, P01 AG005842, P01 AG08291, P30 AG12815, Y1-AG-4553-01 and OGHA 04-064, IAG BSR06-11, R21 AG025169) as well as from various national sources is gratefully acknowledged (see http://www.share-project.org for a full list of funding institutions).

We would like to thank Thorsten Lunau, MA and Natalia Wege, MD, MPH, from the Department of Medical Sociology, University of Duesseldorf, Germany, for fruitful discussions. Moreover, we thank Andrew 'Randy' Pennycott, PhD, London, for English language corrections.

Contributors JDR wrote the first draft. JDR, MW and JS designed the data analysis. MW performed the data analysis. JDR, MW and JS wrote the final draft.

Funding Swiss Paraplegic Research.

Competing interests None.

Patient consent Obtained.

Ethics approval Ethical commissions of the study countries.

Provenance and peer review Not commissioned; externally peer reviewed.

\section{REFERENCES}

1 Vaupel JW. Biodemography of human ageing. Nature 2010;464:536-42.

2 OECD. Sickness, disability and work: breaking the barriers. A synthesis of findings across OECD countries. Paris: OECD, 2010.

3 Avendano M, Mackenbach JP. Life course and labour market exit in 13 European Countries: results from SHARELIFE. In: Börsch-Supan A, Brandt M, Hank K, Schröder M. eds. The individual and the Welfare State. Berlin: Springer, 2011:203-13.

4 Haan P, Myck M. Dynamics of health and labor market risks. J Health Econ 2009:28:1116-25.

5 Huisman M, Kunst AE, Mackenbach JP. Socioeconomic inequalities in morbidity among the elderly; a European overview. Soc Sci Med 2003;57:861-73.

6 Koukouli S, Vlachonikolis IG, Philalithis A. Socio-demographic factors and self-reported functional status: the significance of social support. BMC Health Serv Res 2002;2:20

7 Rautio N, Heikkinen E, Ebrahim S. Socio-economic position and its relationship to physical capacity among elderly people living in Jyvaskyla, Finland: five- and ten-year follow-up studies. Soc Sci Med 2005;60:2405-16.

8 Schoeni RF, Martin LG, Andreski PM, et al. Persistent and growing socioeconomic disparities in disability among the elderly: 1982-2002. Am J Public Health 2005;95:2065-70.

9 Minkler M, Fuller-Thomson E, Guralnik JM. Gradient of disability across the socioeconomic spectrum in the United States. N Engl J Med 2006:355:695-703.

10 Ramsay SE, Whincup PH, Morris RW, et al. Extent of social inequalities in disability in the elderly: results from a population-based study of British men. Ann Epidemiol 2008;18:896-903.

11 Blekesaune M, Solem PE. Working conditions and early retirement—a prospective study of retirement behavior. Research on Aging 2005;27:3-30.

12 Karasek R, Theorell T. Healthy work. New York: Basic Books, 1990

13 Siegrist J. Adverse health effects of high-effort/low-reward conditions. J Occup Health Psychol 1996;1:27-41.

14 WHO. International Classification of Functioning, Disability and Health (ICF). Geneva: WHO Press, 2001.
15 United Nations. Convention on the rights of persons with disabilities. New York, 2006.

16 WHO, World Bank. World report on disability. Geneva: WHO Press, 2011.

17 Dale C, Prieto-Merino D, Kuper $\mathrm{H}$, et al. Modelling the association of disability according to the WHO International Classification of Functioning, Disability and Health (ICF) with mortality in the British Women's Heart and Health Study. J Epidemiol Community Health 2012;66:170-5.

18 Börsch-Supan A, Hank K, Jürges H. A new comprehensive and international view on ageing: introducing the 'Survey of Health, Ageing and Retirement in Europe'. Eur J Ageing 2005;2:245-53.

19 Börsch-Supan A, Jürges H. The survey of health, aging and retirement in Europemethodology. Mannheim: Mannheim Research Institute for the Economics of Ageing, 2005

20 Reinhardt J, von Elm E, Fekete $\mathrm{C}$, et al. Social inequalities of functioning and perceived health in Switzerland-a representative cross-sectional analysis. PLoS One 2012; 7:e38782002E.

21 Cieza A, Geyh S, Chatterii S, et al. ICF linking rules: an update based on lessons learned. J Rehabil Med 2005;37:212-18.

22 Kolenikov S. Confirmatory factor analysis using confa. The Stata Journal 2009;9:329-73.

23 Karasek R, Brisson C, Kawakami N, et al. The Job Content Questionnaire (JCQ): an instrument for internationally comparative assessments of psychosocial job characteristics. J Occup Health Psychol 1998;3:322-55.

24 Siegrist J, Starke D, Chandola T, et al. The measurement of effort-reward imbalance at work: European comparisons. Soc Sci Med 2004:58:1483-99.

25 Marmot M, Siegrist J, Theorell T. Health and the psychosocial environment at work. In: Marmot M, Wilkinson RG. eds. Social determinants of health. 2nd edn. Oxford: Oxford Univ. Press, 2006:97-130.

26 Nunnally JC, Bernstein IH. Psychometric theory. New York: McGraw-Hill, 1994.

27 Paccagnella O, Weber G. Income imputation. In: Börsch-Supan A, Brugiavini A, Jürges $\mathrm{H}$, Mackenbach JP, Siegrist J, Weber G. eds. Health, ageing and retirement in Europe. First results from the survey of health, ageing and retirement in Europe. Mannheim: Mannheim Research Institute for the Economics of Ageing, 2005:357-8.

28 Lynch J, Kaplan G. Socioeconomic factors. In: Kawachi I, Berkman L. eds. Social epidemiology. New York: Oxford University Press, 2000:13-35.

29 Rabe-Hesketh S, Skrondal A. Multilevel and longitudinal modeling using Stata. Lakeway Drive (Texas): Stata Press, 2005.

30 Finkel SE. Causal analysis with panel data. Thousand Oaks, CA: Sage, 1995.

31 Li J, Yang W, Cho SI. Gender differences in job strain, effort-reward imbalance, and health functioning among Chinese physicians. Soc Sci Med 2006; 62:1066-77.

32 Stansfeld SA, Bosma $\mathrm{H}$, Hemingway $\mathrm{H}$, et al. Psychosocial work characteristics and social support as predictors of SF-36 health functioning: the Whitehall II study. Psychosom Med 1998:60:247-55.

33 Kuper $\mathrm{H}$, Singh-Manoux A, Siegrist J, et al. When reciprocity fails: effort-reward imbalance in relation to coronary heart disease and health functioning within the Whitehall II study. Occup Environ Med 2002;59:777-84.

34 Ware JE Jr, Sherbourne CD. The MOS 36-item short-form health survey (SF-36). I. Conceptual framework and item selection. Med Care 1992;30:473-83.

35 Schröder M. Attrition. In: Börsch-Supan A, Brugiavini A, Jürges H, Mackenbach J, Siegrist J, Weber G. eds. (Hrsq.): Health, ageing and retirement in Europe (20042007). Starting the longitudinal dimension. Mannheim: Mannheim Research Institute for the Economics of Ageing, 2008:S.325-30.

36 van Oorschot W, Hvinden B. Disability policies in European countries. Tilburg: Kluwer Law International, 2001

37 Siegrist J, Wahrendorf M. Quality of work, health, and retirement. Lancet 2009:374:1872-3.

38 Kivimaki $M$, Virtanen $M$, Elovainio $M$, et al. Work stress in the etiology of coronary heart disease-a meta-analysis. Scand J Work Environ Health 2006;32:431-42.

39 Stansfeld S, Candy B. Psychosocial work environment and mental health-a meta-analytic review. Scand J Work Environ Health 2006;32:443-62. 